\title{
artigo
}

Azevedo, S.F.; Firmino dos Santos, L.S.; Rampini, R.R.; Ferreira da Silva, A.C.F.; Ferreira dos Santos, M.; Bertholy, M.J.B.;

Sindrome de Sturge-Weber: A importância do cuidado integral na assistência ao adolescente portador

\section{Síndrome de Sturge-Weber: A importância do cuidado integral na assistência ao adolescente portador}

\author{
Sturge-Weber Syndrome: The importance of comprehensive care in assisting adolescent patients \\ Síndrome de Sturge-Weber: la importancia de la atención integral en la asistencia a pacientes adolescentes
}

\begin{abstract}
RESUMO
Objetivos: Descrever a experiência vivenciada por acadêmicos de enfermagem durante o atendimento ao adolescente com Síndrome de Sturge-Weber e a importância do cuidado integral. Método: Trata-se de uma pesquisa descritiva, exploratória, com abordagem qualitativa, do tipo relato de experiência, realizada em um Ambulatório Escola na Região Serrana do Estado do Rio de Janeiro, no mês de junho de 2019. Resultado: BHV, 13 anos, sexo masculino, cursando $4^{\circ}$ ano do Ensino Fundamental, portador da Síndrome de Sturge Weber, menciona que há muito tempo não realizava consulta em um serviço de saúde e exames de rotina. Apresentava, "mancha na face", característica da Síndrome. Possui glaucoma e utiliza Fenobarbital devido à crise convulsiva. Apresenta receio devido "a mancha na face". Os dados sinalizam a necessidade de um cuidado integral. Discussão: Por ser uma doença rara, fica notório a necessidade do cuidado integral, devido as manifestações neurológicas, cutâneas, oculares e orais. Nossa conduta proporcionou retornar aos cuidados oftalmológicos, acompanhamento odontologico, avaliação clínica e neurologica. Considerações finais: Foi possível perceber que para atingir a qualidade no cuidado integral na assistência ao adolescente com uma Síndrome rara, como a de Sturge-Weber é de extrema importância o envolvimento de uma equipe multidisciplinar.
\end{abstract}

DESCRITORES: Síndrome de Sturge-Weber; Consulta de Enfermagem; Adolescente; Equipe Multiprofissional.

\section{ABSTRACT}

Objectives: To describe the experience of nursing students during the care of adolescents with Sturge-Weber Syndrome and the importance of comprehensive care. Method: This is a descriptive, exploratory research, with a qualitative approach, of the type of experience report, carried out in a School Clinic in the Mountain Region of the State of Rio de Janeiro, in the month of June 2019. Result: BHV, 13 years old, male, attending 4th year of elementary school, with Sturge Weber Syndrome, mentions that he had not been to a health service and routine exams for a long time. It had a "spot on the face", characteristic of the syndrome. He has glaucoma and uses Phenobarbital due to the seizure. He is afraid because of "the stain on his face". The data indicate the need for comprehensive care. Discourse: As it is a rare disease, the need for comprehensive care is evident, due to neurological, cutaneous, ocular and oral manifestations. Our conduct allowed us to return to ophthalmic care, dental follow-up, clinical and neurological evaluation. Final considerations: It was possible to realize that in order to achieve quality in comprehensive care in the care of adolescents with a rare syndrome, such as that of Sturge-Weber, the involvement of a multidisciplinary team is extremely important.

DESCRIPTORS: Sturge-Weber Syndrome; Nursing Consultation; Adolescent; Multiprofessional Team.

\section{RESUMEN}

Objetivos: Describir la experiencia de los estudiantes de enfermería durante el cuidado de adolescentes con Síndrome de Sturge-Weber y la importancia de la atención integral. Método: Esta es una investigación descriptiva, exploratoria, con un enfoque cualitativo, del tipo de informe de experiencia, realizado en una Clínica Escolar en la Región Montañosa del Estado de Río de Janeiro, en el mes de junio de 2019. Resultado: BHV, 13 años. , hombre, que cursa el cuarto año de la escuela primaria, con el Síndrome de Sturge Weber, menciona que no había estado en un servicio de salud y exámenes de rutina durante mucho tiempo. Tenía una "mancha en la cara", característica del Síndrome. Tiene glaucoma y usa fenobarbital debido a la convulsión. Tiene miedo debido a "la mancha en su rostro". Los datos indican la necesidad de una atención integral. Discurso: como es una enfermedad rara, la necesidad de atención integral es evidente debido a las manifestaciones neurológicas, cutáneas, oculares y orales. Nuestra conducta nos ha permitido volver al cuidado de los ojos, el seguimiento dental, la evaluación clínica y neurológica. Consideraciones finales: fue posible darse cuenta de que para lograr la calidad en la atención integral en la atención de adolescentes con un Síndrome raro, como el de Sturge-Weber, la participación de un equipo multidisciplinario es extremadamente importante.

DESCRIPTORES: Síndrome de Sturge-Weber; Consulta de Enfermería; Adolescente Equipo Multiprofesional.

RECEBIDO EM: 29/01/2020 APROVADO EM: 02/02/2020 


\section{Simone Fátima de Azevedo}

Enfermeira docente no Colégio Santa Catarina - Petrópolis. Preceptora do Módulo de Enfermagem do Adolescente no Ambulatório escola, pela Faculdade Arthur Sá Earp Neto (FASE). Pós-graduada em Enfermagem em Pediatria e Neonatologia. https:// orcid.org/0000-0003-0581-7063

\section{Lívia da Silva Firmino dos Santos}

Coordenadora do Estágio Supervisionado em Rede Ambulatorial do Curso de Enfermagem da Faculdade Arthur Sá Earp Neto FASE no Ambulatório Escola da FASE. Mestre em Ciências do Cuidado em Saúde Pós-graduada em Enfermagem do Trabalho. https://orcid.org/0000-0002-9740-1956

\section{Rafael Ribeiro Rampini}

Graduando em Enfermagem pela Instituição de Ensino Faculdade Arthur Sá Earp Neto FASE. https://orcid.org/0000-00034779-3645

\section{Ana Carolina Ferreira da Silva}

Graduanda em Enfermagem pela Instituição de Ensino Faculdade Arthur Sá Earp Neto FASE. https://orcid.org/0000-00017231-0882

\section{Mariana Ferreira dos Santos}

Graduanda em Enfermagem pela Instituição de Ensino Faculdade Arthur Sá Earp Neto FASE. https://orcid.org/0000-00023764-3865

\section{Márcio José Barbosa Bertholy}

Enfermeiro Graduado em Enfermagem pela Instituição de Ensino Faculdade Arthur Sá Earp Neto FASE. https://orcid.org/00000003-0620-4832

\section{INTRODUÇÃO}

A Síndrome de Sturge-Weber (SSW) é originada a partir de uma alteração genética, por volta da sexta semana de gestação, onde ocorre a mutação do gene GNAQ responsável pelo processo normal de maturação vascular, essa mutação permite que haja uma falha na regressão do plexo venoso cefálico primitivo, interferindo no desenvolvimento normal da face, olhos e cérebro, gerando os principais sinais e sintomas que caracterizam a doença ${ }^{(1,2)}$.

Portanto, caracteriza-se por gerar malformações vasculares a nível cerebral, facial e ocular, a Síndrome de Sturge-Weber é considerada uma doença rara com incidência mundial de um caso em 50.000 indivíduos $^{(3,4)}$.

Uma característica típica desta doença é a mancha vinho do porto, que geralmente surge nos primeiros anos de vida, uma mancha de coloração roxa/avermelhada que apresenta margens bem delimitadas. A mancha tende a localizar-se em áreas inervadas pelo nervo trigêmeo, estendendo-se do ramo oftálmico ao mandibular, de forma unilateral ou bilateral.
Com o passar dos anos a mancha pode aumentar em espessura e intensidade de coloração, gerando inclusive possíveis nódulos angiomatosos ${ }^{(1,5)}$.

O quadro clínico da doença contempla, além da mancha vinho do porto, acometimento ocular e de sistema nervoso central, gerando baixa acuidade visual, hemianopsia, hemangioma escleral, conjuntival ou de coroide, glaucoma, e hemangioma leptomeníngeo que pode ocasionar hipertrofia do parênquima cerebral e calcificação cortical e subcortical propiciando convulsões, atraso no desenvolvimento, deficiência intelectual, dificuldade de aprendizagem, hemiparesia e cefaleia ${ }^{(1,2)}$.

A síndrome pode ainda causar alterações pela cavidade oral por meio de angiomatoses, provocando hiperplasia gengival, macroglossia, e outras alterações ortodônticas que aumentam o risco de sangramento durante procedimentos odontológicos ou intubações ${ }^{(5,6)}$.

A Síndrome de Sturge-Weber pode ser classificada em três tipos, segundo a escala de Roach: Tipo I, caracterizada pela presença da mancha vinho do porto e hemangioma leptomeníngeo, com ou sem glau- coma; Tipo II, com a presença da mancha vinho do porto e glaucoma pré-existente; E Tipo III, com hemangioma leptomeníngeo com ou sem a presença de glaucoma ${ }^{(2)}$.

Tratando-se de uma doença que envolve múltiplas variáveis, o cuidado integral ao paciente portador da síndrome é de fundamental importância. A integralidade do cuidado perpassa a condição de se tratar exclusivamente de um princípio do SUS, sua dimensão é vista como um valor expresso por profissionais de saúde em sua prática assistencial através da interação entre o profissional e o paciente ${ }^{(7)}$.

Um dos atores essenciais na implementação do princípio da integralidade em sua prática profissional são os profissionais da enfermagem, que na assistência prestada ao indivíduo sadio ou enfermo contribuem para a avaliação e propostas de estratégias de cuidado à saúde de crianças e adolescentes nos diversos níveis de assistência à saúde ${ }^{(8)}$.

Neste sentido, cabe mencionar que a adolescência é um período do desenvolvimento que ocorre entre a infância e a fase adulta, esta fase é marcada por mudanças corporais, sociais, emocionais e intelec- 


\section{artigo}

Azevedo, S.F.; Firmino dos Santos, L.S.; Rampini, R.R.; Ferreira da Silva, A.C.F.; Ferreira dos Santos, M.; Bertholy, M.J.B.;

Sindrome de Sturge-Weber: A importância do cuidado integral na assistência ao adolescente portador

tuais no decorrer dos 10 aos 19 anos de idade, tendo características complexas, onde ocorrem inúmeros fenômenos como a busca de uma identidade, a valorização dos grupos sociais e possível afastamento dos valores familiares, crises religiosas, desenvolvimento da sexualidade, para assim construir a sua própria personalidade ${ }^{(9,10)}$.

Através deste estudo pretende-se responder a seguinte questão norteadora: Como prestar o cuidado integral na assistência ao adolescente portador Síndrome de Sturge-Weber?

De acordo com o já mencionado, o objetivo do presente estudo é descrever a experiência vivenciada por acadêmicos de enfermagem durante o atendimento ao adolescente com Síndrome de Sturge-Weber e a importância do cuidado integral.

\section{METODOLOGIA}

Trata-se de uma pesquisa descritiva, exploratória, com abordagem qualitativa, do tipo relato de experiência, realizada em um Ambulatório Escola de uma Instituição de Ensino Superior privada, na Região Serrana do Estado do Rio de Janeiro, no mês de junho de 2019, especificamente no dia 12/06/2019.

$\mathrm{O}$ relato foi baseado na experiência dos discentes, do $7^{\circ}$ e $9^{\circ}$ Período, do Curso de Bacharelado em Enfermagem, enquanto cursavam a disciplina "Estágio Supervisionado em Rede Ambulatorial" no Módulo de Enfermagem Saúde do Adolescente, sob a supervisão da Enfermeira Preceptora.

E sua vivência durante o atendimento ao paciente adolescente com Síndrome de Sturge-Weber, esclarecendo dúvidas e atuando como mediador durante esse processo para ofertar um cuidado integral, acionando a equipe multiprofissional para atuar na assistência ao adolescente portador dessa síndrome rara.

O serviço de enfermagem do adolescente possui parceria com diversas instituições de ensino, e uma delas é uma Escola Municipal próximo à Unidade de Saúde, onde a pedido da diretora, são realizadas atividades em educação em saúde e os alunos direcionados para as consultas no ambulatório. As atividades realizadas por acadêmicos de enfermagem, do Módulo de Enfermagem do Adolescente, são supervisionadas pela Enfermeira Preceptora, e os alunos beneficiados são das turmas do $4^{\circ}$ e $5^{\circ}$ ano do Ensino Fundamental.

Cabe ressaltar, que por se tratar de um relato de experiência não houve necessidade de submissão ao Comitê de Ética em Pesquisa, porém foram respeitados pelos autores os aspectos éticos na elaboração do mesmo.

\section{RELATO DA EXPERIÊNCIA}

$\mathrm{Na}$ consulta de Enfermagem, BHV, adolescente, 13 anos, sexo masculino, cursando $4^{\circ}$ ano do Ensino Fundamental, compareceu acompanhado pelo pai, encaminhado pela Escola Municipal onde os acadêmicos de enfermagem realizam ações educativas em saúde.

No primeiro momento, durante o acolhimento a acadêmica observou que o adolescente apresentava, "mancha na face". Pai relatou que o filho é portador da Síndrome de Sturge Weber e há muito tempo não realizava consulta em um serviço de saúde e exames de rotina.

Durante a consulta de enfermagem do adolescente, os acadêmicos realizam uma abordagem com visão global, sendo composta das seguintes etapas: anamnese, exame físico, orientações e encaminhamentos, se necessário. No momento da anamnese é preenchido o instrumento de primeira consulta, onde adolescente e o familiar presente respondem a coleta dos dados: identificação, antecedentes pessoais, antecedentes familiares, dados familiares, condições de habitação, hábitos alimentares/ingesta hídrica e eliminações vesico intestinais.

Neste momento, os acadêmicos conseguiram identificar que o adolescente é portador de glaucoma e utiliza Fenobarbital. Identificaram, ainda, que o adolescente foi internado logo após o nascimento devido à Síndrome de Sturge-Weber e aos 03 anos foi novamente internado, devido à crise convulsiva, permanecendo sob os cuidados hospitalares durante 23 dias. Foi neste período que o fenobarbital $40 \mathrm{mg}(2$ vezes ao dia) foi prescrito, e após fazer uso da medicação não houveram novos episódios de convulsão. Faz uso de colírio desde os dois anos de idade, atualmente utiliza o Cosopt (uma gota em cada olho), devido glaucoma em olho esquerdo.

No segundo momento da anamnese, ainda sob supervisão do preceptor, os acadêmicos ficam a sós com o adolescente, pois é o momento mais importante da consulta, uma vez que é a oportunidade para o adolescente se sentir seguro para expor questões sigilosas e para o aluno reforçar o vínculo criado na etapa anterior. Nesse momento o aluno questionou sobre: questões da puberdade (gineco-urológico) e sexualidade, autoimagem e aceitação corporal, processos psicoemocionais, espaço escolar e educação, trabalho, vida social, hábitos de vida, acuidade visual e auditiva e caderneta de vacina.

O que chama atenção dos acadêmicos, até mesmo devido as questóes relacionadas à síndrome, é que o paciente nega dificuldade de visualizar o quadro durante a aula e dificuldade auditiva, porém, relata três reprovações nos anos escolares. Refere gostar de estudar e ser assíduo as aulas. Apresenta dificuldade grave de leitura e pouca escrita, utiliza palavras curtas e simples. Apesar da faixa etária ainda está iniciando a pintura de figuras dentro do limite interno da imagem e relata sentir mais segurança e firmeza quando utiliza caneta. Apresenta-se ativo, comunicativo, extrovertido, relata boa interação com os colegas da escola e da rua onde reside. Joga futebol aos sábados e brinca com videogame. Possui cartão de vacina desatualizado. Apresenta autoestima elevada e apesar de estar animado para começar a ir de ônibus para sua nova escola no ano de 2020 , tem receio devido "a mancha na face" e ter que explicar sobre sua patologia aos novos colegas de escola.

Os acadêmicos sinalizam preocupação com o fato do paciente estar até então em um ambiente seguro e já conhecido, onde não havia problema algum com as características da doença que é a mancha do vinho do porto em face, mas a escola nova poderá trazer desconforto devidos os 
olhares dos colegas.

Ao exame físico apresentou os seguintes parâmetros: PESO: $41,200 \mathrm{~kg}$; ALTURA:1,51m; IMC: 18,07; TAX: 35.9; FR: $16 \mathrm{rpm}$; FC: $76 \mathrm{bpm}$; PA: 120/60 mmHg; Estagiamento de Tanner: P2 e G1. Os dados foram classificados como adequados para a idade, segundo a Caderneta do adolescente do Ministério da Saúde ${ }^{(11)}$.

Integridade da pele sem alterações e coloração da pele conforme traços da Síndrome; a palpação superficial e profunda em abdome sem alterações ou massas palpáveis; observado hiperpigmentação em quadrantes superiores e quadrantes inferiores, que se estende até os membros inferiores onde a coloração se normaliza.

Ao término da consulta, os acadêmicos destacaram como maior risco os seguintes diagnósticos de enfermagem: 1-Risco de baixa autoestima situacional relacionado à alteração da imagem corporal. 2-Dentição prejudicada caracterizado por cáries dentais relacionado à higiene oral inadequada.

Seguiram a consulta com as seguintes orientações, quanto a: atualização da caderneta de vacinação; importância da higiene oral; importância de se dedicar aos estudos; perspectiva para o futuro; bullying; acerca da ansiedade sobre a nova escola e incentivado a permanecer com autoestima elevada; ao não envolvimento com drogas lícitas e ilícitas;

Para fechar a consulta e atender a demanda do adolescente, segundo o diagnóstico de enfermagem encontrado, os acadêmicos e a preceptora optaram pela seguinte conduta: encaminhamentos para os seguintes serviços: oftalmologia; neuropediatria; clínica médica; odontologia; sala de vacina para realizar a administração da primeira dose da vacina HPV e meningocócica; solicitação de exames laboratoriais da rotina do adolescente e agendamento de consulta de retorno com a enfermagem.

A preceptora e alunos entendendo que para prestar o cuidado integral na assistência ao adolescente portador da Síndrome de Sturge-Weber seria necessário, não somente as orientações sobre os cuidados gerais de saúde na fase da adolescência, mas também a participação de uma equipe multidisciplinar, para assim trazer resolutividade às necessidades do paciente e consequentemente evitar complicações futuras. Dessa forma os profissionais de cada especialidade foram contatados e após passagem do caso conseguiu-se os seguintes desfechos:

- Oftalmologista - atendido em 19/06/2019, adolescente chegou acompanhado "da mãe do coração" (SIC), refere histórico de glaucoma, mencionou que fazia acompanhamento no Rio, na UFRJ. No momento da consulta queixou-se de dificuldade de ler ao longe. Em uso diário de colírio desde os 02 anos de idade. Ao exame físico, evidenciado, hemangioma em hemiface D (principalmente) e E. Na avalição oftalmológica: $\mathrm{AV}: \mathrm{OD}=\mathrm{NPL}$ e $\mathrm{OE}=20 / 20 \mathrm{ct}(+1,00 \mathrm{esf} / 1,50 \mathrm{cil} \mathrm{a}$ $\left.170^{\circ}\right)$. Bio: Od: catarata branca, sinéquias posteriores; ceratopatia em faixa. OE: sem alterações; $\mathrm{PIO}=30 / 30, \mathrm{FO}$ : OD: indevassável, OE: DO esc 0,5 e macula sem alterações, vasos regulares, retina aplicada sem lesões retinianas evidentes. Conduta: Prescrito novo colírio em OE, e encaminho para consulta no Serviço de Oftalmologia do Hospital Universitário Pedro Ernesto - HUPE (UERJ);

- Neuropediatra - atendido em 26/06/2019 às 10:30h, refere fazer uso de fenobarbital $40 \mathrm{mg}$ (2 vezes ao dia); última crise convulsiva aos 03 anos, difícil controle; episódio de hemiparesia direita súbita; último exame de imagem aos 03 anos. Ao exame físico: hemangioma plano de face com predomínio a $\mathrm{D}$, aumento de partes moles hemiface $\mathrm{D}$ e língua; sem déficit motor focal, mas pouca motricidade fina, inclusive grafismo. Conduta: solicitado ressonância magnética cardíaca para avaliação de más formações vasculares associadas; USG abdome total para avaliação de possíveis outros hemangiomas; aumentada a dose de fenobarbital para $100 \mathrm{mg}$; aguardando avaliação do nível sérico sanguíneo;

- Clínica médica - atendido em
26/06/2019, às 13:00h, acompanhado pela madrasta, sem queixas, já em acompanhamento com a neuropediatra. Realizada avaliação clínica sem alterações. Conduta: solicitado exames laboratoriais e no aguardo de exames já solicitado pela neuropediatra. Retorno após 03 meses;

- Odontologia - atendido em 28/06/2019, queixa principal do acompanhante "ele não gosta de escovar os dentes, então a situação está ruim. Tem que dar uma olhadinha”. Adolescente nunca foi ao dentista não sendo colaborativo com a higiene oral. Paciente encaminhado pela enfermagem devido a presença de lesões orais. Observou-se acúmulo de placa nas superfícies da maioria de seus dentes, com cáries proximais (entre faces vizinhas) e nas ocluais. A destruição das superfícies dentárias pelo processo carioso era acentuada nos seus primeiros molares, que já não apresentavam coroas viáveis. Foi realizada uma radiografia panorâmica que não evidenciou lesões intraósseas. Sua dentição era composta de dentes decíduos e permanentes, alguns ainda em fase de erupção. Há necessidade de uma adequação do meio oral, com eliminação de focos infecciosos, limpeza das cáries e colocação de material restaurador. Ainda, a remoção de alguns dentes, inclusive permanentes, se faz necessária, o que demandará um planejamento de reabilitação para manutenção da capacidade mastigatória. É importante salientar que é exuberante a presença de angiomas intraorais, inclusive em áreas adjacentes aos dentes que deverão ser removidos. Observado aumento de volume em lábio inferior, linfonodo cervical anterior E, aumentado/dolorido, consistência firme e móvel. Nesta primeira consulta, foi realizada limpeza e aplicação de flúor. Desta forma, é essencial traçar um plano de tratamento considerando a necessidade de intervenções em ambiente hospitalar. O paciente foi remarcado devendo ser acompanhado no serviço de odontologia. 
Enquanto acadêmicos, foi possível perceber que um dos fatores essenciais para o cuidado integral na assistência ao adolescente com a síndrome foi o fato da unidade de saúde, ambulatório escola, ser pertencente a uma Faculdade privada com compromisso com ensino e que possui convênio com o Sistema Único de Saúde (SUS), portando um campo de prática para os estágios dos seus cursos de graduação, por isso foi capaz de atender a demanda em um prazo extremamente rápido respeitando os princípios fundamentais do SUS: a universalidade, a integralidade e a equidade.

$\mathrm{O}$ que nos faz pensar que talvez em outro serviço, onde se dependa exclusivamente do SUS, esses agendamentos com as especialidades não aconteçam com tamanha agilidade.

Desta forma, os resultados deste estudo apontam a grande importância de instituições de saúde que tenham responsabilidade com o ensino/pesquisa, colaborando com a formação de profissionais de saúde para o SUS, proporcionando assim melhoria da qualidade de vida da população assistida.

\section{DISCUSSÃO}

A literatura aponta que com relação a Síndrome de Sturge-Weber, os sintomas devem ser abordados separadamente, uma vez que não existe tratamento específico $^{(10)}$. O suporte ao paciente com a síndrome envolve várias questões, inclusive as psicossociais, uma vez que exercem importante papel no acompanhamento do paciente e de seus familiares. Principalmente por se tratar de um adolescente que para o controle das comorbidades necessita do apoio da família a fim de dar continuidade do tratamento.

Durante a adolescência é possível que a saúde seja comprometida de maneira irreversível, portanto cabe aos profissionais de saúde maior atenção nessa fase da vida, a fim de que se reconheça as demandas dessa população e se possa intervir oportunamente, sendo assim é extremamente importante que adolescente acesse os serviços de saúde ${ }^{(12)}$. Percebemos ao acompa-

\section{As lesões cutâneas da} síndrome geralmen-

te não necessitam

de terapia estética

precoce, pois existe

a possibilidade dessa

mancha ser disfarça-

da com a utilização

de cosméticos ou ser

tratada permanente-

mente por terapia a

laser ${ }^{(10)}$. Nosso pacien-

te encontra-se em um

momento de desafio

em uma escola nova, e

o caso dele nos revela

que conviver com uma

alteração na pigmenta-

ção, textura ou forma-

to da pele pode trazer

consequências, princi-

palmente nesta fase de

descobertas, vaidade e

transformação que é a

adolescência. nhar esse caso que por se tratar de uma doença rara e, principalmente por acometer um adolescente, fica notório a necessidade de um olhar diferenciado, uma vez que a Síndrome de Sturge-Weber apresenta manifestações neurológicas, cutâneas, oculares e orais que podem (ou não) estar associadas entre si.

Segundo autores ${ }^{(13)}$, com relação ao acesso do adolescente aos serviços de saúde, a procura é espontânea ou ocorre por meio de encaminhamentos da rede de saúde, assistência social ou da escola. O serviço de saúde deve interagir com a escola, e na nossa experiência o fato da unidade de saúde, Ambulatório Escola, possuir parceria com várias escolas nos permitiu conhecer esse adolescente em seu ambiente escolar por meio de atividades educativas e, posteriormente, acompanhá-lo da melhor forma no consultório.

As lesões cutâneas da síndrome geralmente não necessitam de terapia estética precoce, pois existe a possibilidade dessa mancha ser disfarçada com a utilização de cosméticos ou ser tratada permanentemente por terapia a laser ${ }^{(10)}$. Nosso paciente encontra-se em um momento de desafio em uma escola nova, e o caso dele nos revela que conviver com uma alteração na pigmentação, textura ou formato da pele pode trazer consequências, principalmente nesta fase de descobertas, vaidade e transformação que é a adolescência.

As complicações oculares da síndrome se manifestam, como glaucoma e malformações vasculares da conjuntiva, episclera, coróide e retina ${ }^{(14)}$. O acompanhamento anual para o glaucoma, por meio de fundoscopia e tonometria, é recomendado para todos os pacientes com Síndrome de Sturge-Weber ${ }^{(10)}$. Neste caso, foi possível que o paciente em questão retornasse aos cuidados oftalmológicos, sinalizando que nossa atuação enquanto acadêmicos foi de tamanha importância ao identificar a necessidade e realizar o encaminhamento pós contato com o profissional.

O manejo da Síndrome de Sturge-Weber envolve o envolvimento multidisciplinar, sendo o neurologista um dos profissionais importantes para o reconhe- 
cimento precoce e controle das crises convulsivas e prescrição de drogas antiepilépticas para prevenir convulsões e, portanto, evitar lesão neurológica progressiva ${ }^{(15)}$. Sendo assim, o desfecho da nossa consulta de enfermagem ao encaminhar o paciente para a Neuropediatra, que alterou a dose do fenobarbital e solicitou exames específicos de imagem, nos dá a satisfação de ter colaborado com a promoção da saúde deste adolescente.

Com relação às manifestações orais, elas estão presentes em aproximadamente $38 \%$ dos casos e podem envolver lesão hemangiomatosa no lábio, mucosa oral, gengiva, língua e região palatina ${ }^{(14)}$.

No caso do nosso paciente, uma manutenção deficiente da higiene bucal foi observada durante o acompanhamento odontológico, essa deficiencia e citada como consequencia da Síndrome devido ao funcionamento cognitivo levemente desafiado, que pode intensificar a inflamação e a hiperplasia gengival ${ }^{(14)}$.

O acompanhamento odontológico para o adolescente com a Síndrome de Sturge-Weber é fundamental e, em alguns casos, há necessidade de realizar procedimentos apenas em unidades hospitalares devido ao risco de sangramento. A reabilitação dentária desses pacientes é um processo complexo ${ }^{(14)}$, daí a importancia do nosso paciente ser acompanhado para realizar extrações dentarias e permanecer sob avaliação periódica.

Fica evidente, durante nosso relato de experiência, a necessidade da atuação em equipe, pois o engajamento e envolvimento entre os profissionais das diversas áreas contribui para melhor assistir o adolescente, devido à variedade de alterações encontradas na Síndrome de Sturge-Weber, sendo assim, é de fundamental importância a avaliação periódica e acompanhamento dos casos por parte de uma equipe interdisciplinar ${ }^{(16)}$.

\section{CONCLUSÃO}

Os achados em nosso paciente foram semelhantes aos mencionados na literatura e a assistência realizada conforme os apontamentos descritos. Foi possível evidenciar que para prestar o cuidado integral na assistência ao adolescente portador de uma síndrome rara, como a de Sturge-Weber é de extrema importância o envolvimento de uma equipe multidisciplinar.

Essa experiência, enquanto acadêmicos de enfermagem, nos permitiu fazer várias contribuições devido um olhar integral para esse indivíduo, que terá que conviver e se adaptar às diversas alterações físicas e emocionais inerentes da sua patologia. A possibilidade de ter uma equipe multidisciplinar em parceria com o serviço de enfermagem do adolescente nos possibilitou avanços significativos na integralidade da assistência, sendo essa uma característica da unidade de saúde envolvida.

Espera-se que este relato de experiência contribua auxiliando muitos profissionais durante o seu processo de trabalho e aos acadêmicos um olhar integral ao paciente durante sua aprendizagem.

\section{REFERÊNCIAS}

1. Londono GW, Pierre JJ, Calderón M. Síndrome de Sturge-Weber: reporte de um caso. Revista Médico Científica. 2019 jun; 31:26-31.

2. Arango JC, Villada HC. Síndrome de Sturge Weber. Neurociencias Journal. 2017; 24(3):225-230.

3. Lizano MH. Síndrome de Sturge Weber. Revista Auspiciada por el Hospital Dr. Rafael Ángel Calderón Guardia. 2015; 82(617).

4. Canelas C, Lima J. Sindrome de Sturge-Weber-: Os síndromes raros também existem. Galicia Clínica. 2015; 76(4):186.

5. Pérez Al, Maraña, et al. Análisis del síndrome de Sturge-Weber: estudio retrospectivo de múltiples variables asociadas. Neurología. 2017; 32(6):363-370.

6. Santos DMM, et al. Exodontia em paciente com síndrome de Sturge-Weber: relato de caso. Revista da AcBO. 2016; 5(1).

7. Santos ATS, et al. Integralidade do cuidado na formação do enfermeiro: visões e vivências do acadêmico de enfermagem. Enfermagem em Foco. 2019; 10(1).

8. Jorge $\mathrm{KJ}$, et al. Integralidade e formação do enfermeiro para o cuidado a criança e adolescente. Varia Scientia-Ciências da Saúde. 2016; 2(2):127-137.

9. Biffi D, Mello MFR, Ribeiro VR. Acolhimento de enfermagem à saúde do adolescente em uma estratégia de saúde da família.

\section{8; 3(1).}

10. Oliveira MR. Estudos sobre a adolescência e os conflitos sociofamiliares. Psicologia.pt. 2018.

11. Ministério da Saúde, Secretaria de Atenção à Saúde (BR). Caderneta de saúde do adolescente. 2. ed. Brasília, DF: Ministério da Saúde, 2010. 52 p. (Série F. Comunicação e Educação em Saúde).

12. Martins MMF, Aquino R, Pamponet ML, Pinto Junior EP, Amorim LDAF. Acesso aos serviços de atenção primária à saúde por adolescentes e jovens em um município do Estado da Bahia, Brasil. Cad. Saúde Pública. 2019; 35(1):e00044718.

13. Silva JF, Matsukura TS, Ferigato SH, Cid MFB. Adolescência e saúde mental: a perspectiva de profissionais da Atenção Básica em Saúde. Interface (Botucatu). 2019; 23:e18063.

14. Godge P, Sharma S, Yadav M, Patil P, Kulkarni S. Sturge Weber syndrome: a case report. Rev. odonto ciênc. 2011; 26(4):366369.

15. Wertheimer G. Síndrome de sturge weber. Dr.Pixel. Campinas. 2016.

16. Santos SVP, Cavalheiro LG. Síndrome de Sturge-Weber: relato de caso com base na avaliação fonoaudiológica. Rev. CEFAC. 2010 Feb; 12(1):161-170. 